\title{
Utilizing spatial association analysis to determine the number of multiple grids for multiple-point statistics
}

\author{
Hexiang Bai ${ }^{\mathrm{a}}$, Yong Ge ${ }^{\mathrm{b}, *}$, Gregoire Mariethoz ${ }^{\mathrm{c}}$ \\ a School of Computer and Information Technology, Shanxi University, No. 92 Wuchen Road, Taiyuan, \\ Shanxi 030006, China \\ ${ }^{\mathrm{b}}$ State Key Laboratory of Resources and Environmental Information System, Institute of Geographic \\ Sciences and Natural Resources Research, Chinese Academy of Sciences, A11 Datun Road, Chaoyang \\ District, Beijing 100101, China \\ ${ }^{\mathrm{c}}$ Institute of Earth Surface Dynamics (IDYST), School of Civil and Environmental Engineering, University of \\ Lausanne, Mouline, Geopolis, office 3337, 1015 Lausanne, Switzerland
}

\section{A R T I C L E I N F O}

Article history:

Received 16 September 2015

Accepted 8 June 2016

Available online 18 June 2016

\section{Keywords:}

Multi-point simulation

Multiple grid simulation

Multiple grid number

Join count statistics

Single normal equation simulation

\begin{abstract}
A B S T R A C T
Scale selection is a fundamental issue of spatial analysis. Based on spatial association analysis, this paper proposes a quantitative method for estimating the scale, which is represented using the minimal number of multiple grids in a single normal equation simulation. First, the largest scale structural information that a data template can consider for the finest grid is computed using the distance between the central cell and the border of the data template. The maximum distance at which cells are associated with each other in the training image is then analyzed using join count statistics. Finally, the minimal number of multiple grids is estimated based on the criterion that the data template used on the largest grid should account for the maximum distance between associated cells. The proposed method is validated using two- and three-dimensional experiments. The results show that increasing the number of multiple grids does not significantly improve the simulation quality when the number of multiple grids used is larger than that estimated. A sensitivity analysis demonstrates that the proposed method adapts to the configuration of the data template,
\end{abstract}

Abbreviations: JCS, join count statistic; MPS, multi-point simulation; NJCS, normalized join count statistic; SNESIM, single normal equation simulation.

* Corresponding author.

E-mail addresses: baihx@sxu.edu.cn (H. Bai), gey@lreis.ac.cn (Y. Ge), Gregoire.Mariethoz@unil.ch (G. Mariethoz). 
the geometric structure of the target surface objects, and a rescaled training image if it adequately represents the large-scale structural information of the target surface objects.

(c) 2016 Elsevier B.V. All rights reserved.

\section{Introduction}

Multi-point statistics for geological facies modeling was first proposed in the 1990s (Guardiano and Srivastava, 1993; Journel, 1993). The aim of this method is to overcome the limitations of variogrambased approaches - which can only consider two-point correlations - by simultaneously computing correlations for not only two points but also more than two points. Currently, there are many effective algorithms that perform multi-point simulations (MPS) based on multi-point statistics (Li et al., 2005; Strebelle, 2002; Tahmasebi et al., 2012; Zhang et al., 2006). MPS is instructive in describing objects with complex geometries. Compared with variogram-based approaches, it is more effective in capturing complex structure information over different scales. Of these algorithms, the single normal equation simulation (SNESIM) (Boucher, 2009; Cears and Zhang, 2004; Mirowski et al., 2009; Strebelle, 2002; Zhang et al., 2005) was the first to reduce the CPU and memory demands to an acceptable level using multiple grid simulation techniques and a search tree data structure. Although newer implementations exist, SNESIM is still the most widely used MPS method, largely because of its userfriendly interface (Remy et al., 2009). Mariethoz and Lefebvre (2014) suggested combining MPS with texture synthesis. MPS has been extensively and successfully used to model geographical phenomena that have complex spatial structures. For example, it has been applied to model fluid flow behavior (Hajizadeh et al., 2011; Okabe and Blunt, 2004), perform super-resolution mapping (Boucher, 2008; Ge, 2013; Jha et al., 2013; Mariethoz et al., 2011), classify remotely sensed imagery (Ge and Bai, 2011; Tang et al., 2013), map fossil ice-wedge polygons (Meerschman et al., 2014), and reconstruct sandstone (Xu et al., 2012). Mahmud et al. (2015) proposed the use of MPS for integrating different hydraulic conductivity measurements taken at different scales.

An important issue with the use of MPS is that the underlying spatial model does not only depend on the training image used, but also on a series of algorithmic parameters. In the case of several simulation methods, the parameter defining the number of multiple grids is critical. The parameter number of multiple grids refers to the number of sub-grids that the MPS used to generate simulations. The larger the number of multiple grids used, the larger the scale of the structural information that the simulation takes into account. Earth surface phenomena and processes have obvious multi-scale characteristics. How to reproduce the characteristics of different scales for target surface objects is an important issue. SNESIM uses multiple grids to reproduce large-scale structural information, thus efficiently improving the simulation quality. This has been adopted by several MPS algorithms, such as FILTERSIM (Zhang et al., 2006) and IMPALA (Straubhaar et al., 2011). Sensitivity analyses of the number of multiple grids (Liu, 2006; Strebelle, 2000) have revealed that it is critical to achieving acceptable simulation results. For example, if the number of multiple grids is too small, only smallscale structures of less than the size of the currently used data template can be captured, and the results will be unacceptable (Liu, 2006). A multiple grid number that is too large may not improve the simulation quality, and will waste computational resources (Liu, 2006). These results provide only empirical guidelines for determining the appropriate number of multiple grids.

Although the use of a conservatively large number of multiple grids is a safe approach for generating simulation results and only takes a little more time for simulation, this will indeed limit the application of MPS. MPS has been used in many applications to characterize and model the complex geometric structures of earth surface objects, for example for the classification of remotely sensed imagery (Ge and Bai, 2011; Tang et al., 2013) and super-resolution mapping (Boucher, 2008; Ge, 2013; Jha et al., 2013; Mariethoz et al., 2011). If there are numerous data (e.g. a collection of remotely sensed images) to be handled and many different training images for different objects are used for these 
data, it is labor intensive to choose a "conservative" number of multiple grids for all training images as these images may come from different sources and have different sizes. For example, historical remote sensing imagery contains patterns and objects that can be used as training images. These training images are useful for improving classification accuracy for future remotely sensed imagery. The structure characteristics of the same object may differ in different places or different times. The minimal multiple grid numbers of training images containing these objects differ greatly and it is hard to determine the conservative multiple grid number for each training image manually. Meanwhile, in this scenario, automatically handling all data can speed up the production and commercial application of the data product and reduce the risk for human error. Accordingly, the automatic selection of the number of multiple grids to be used is needed for real life applications.

Currently, there is no quantitative criterion to judge whether the number of multiple grids is conservatively large enough. If the number of multiple grid is set as large as possible, for example with the largest enlarged data template covering the whole training image, two problems may arise. First, training images for multipoint geostatistical methods must satisfy stationarity and ergodicity constraints (Cears and Zhang, 2004). When the number of multiple grids is too large, it is no longer reasonable to assume that all possible patterns corresponding to the enlarged data template are available in the training image, i.e., the training image does not satisfy the ergodicity constraint at this scale. Second, as there are only few patterns corresponding to the enlarged data template in the training image, there may not be a large enough number of replicates at this scale. This can result in an excessive replication of the patterns in the training image (known as verbatim copy). Accordingly, an optimal value for the number of multiple-grids is needed.

To illustrate this, Fig. 1 showsthe mean and variance of 100 simulations using the training image in Fig. 4(a) and the data template in Fig. 2(c) conditioned to 10 hard data points. Purple circles in the figure represents hard data points labeled ' 1 ' and blue circles in the figure represents hard data points labeled ' 0 '. The size of the simulation results is $200 \times 200$ pixels. The maximum number of conditioning data is set to 32 , the minimum number of replicas (mincd) is set to 20 , and the marginal probabilities of the black and white classes are set to 0.3 and 0.7 , respectively. Fig. 1(a)-(c) shows the mean and variance of 100 simulation results using the number of multiple grids 1,4 , and 8 , respectively.

When the number of multiple grids is small, the white areas (with a variance close to zero) are mainly distributed around hard data points. However, when the multiple grid number is too large, for example 8 , it is found that the areas of low variance (in white) become larger, and distributed not only around hard data but also around many locations that are far from the hard data. A reasonable explanation is that the training image did not satisfy the ergodicity constraint at large scales, and that this effect is caused by the excessive number of multiple grids, resulting in verbatim copy of the training image.

Recently, Kolbjørnsen et al. (2014) developed a technique for quantitatively determining the optimal number of multiple grids. They used the lag of the correlation functions to evaluate a starting grid level (which is similar to the number of multiple grids in SNESIM) for multiple grid-based Markov mesh facies modeling. This was the first effort at automatic determination of the number of multiple grids. According to their experiments, their method can effectively determine the starting grid level. However, they also pointed out in their paper that a robust quantitative method of estimating the starting values for an arbitrary training image is needed (Kolbjørnsen et al., 2014). Meanwhile, Kolbjørnsen et al. (2014) did not discuss if the starting grid found is the minimum required to generate simulation results with acceptable quality.

In addition to the close relation to simulation quality, scale selection is also a fundamental step in the determination of other MPS parameters. For example, selecting and ranking training images in terms of the data at hand depend on the user-defined scale (Pérez et al., 2014). Pérez et al. (2014) presented a method that can effectively and efficiently select the most appropriate training image from a series of candidate images. However, if only small-scale structures in the candidate training images are used, then this method may not rank the candidate images correctly. Therefore, it is necessary to develop effective methods for quantitatively determining the relevant spatial structural scale. In many MPS algorithms, the spatial structural scale is set via the number of multiple grids. 

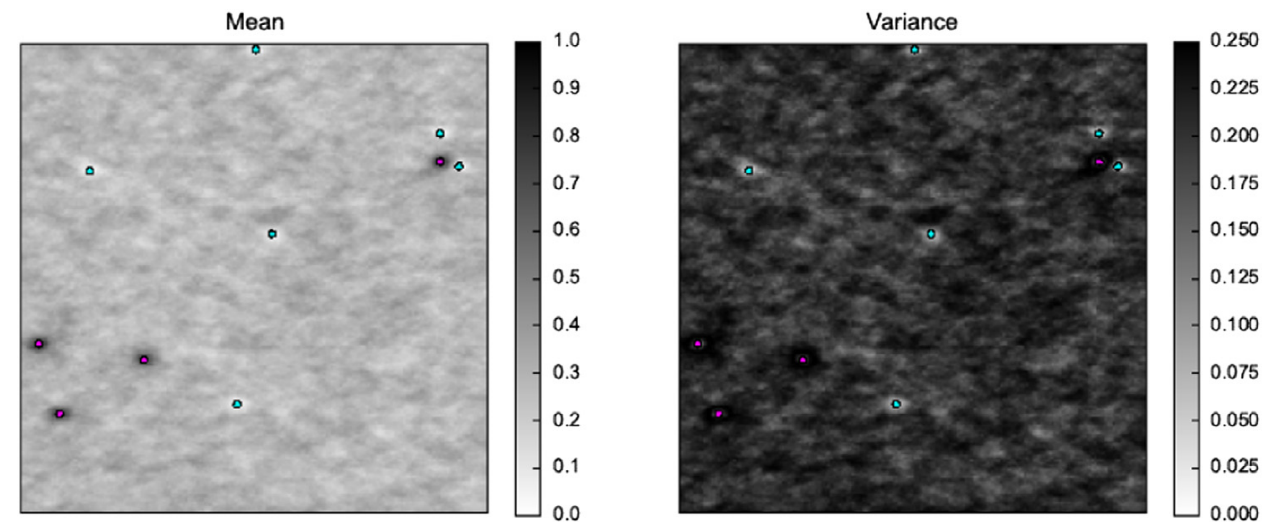

(a) $G=1$.
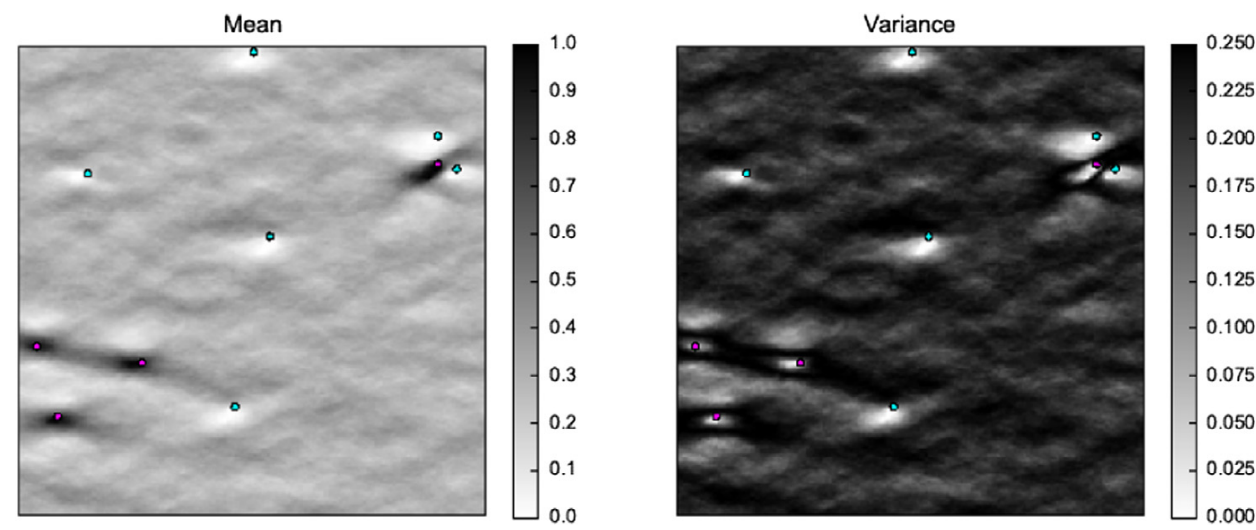

(b) $G=4$
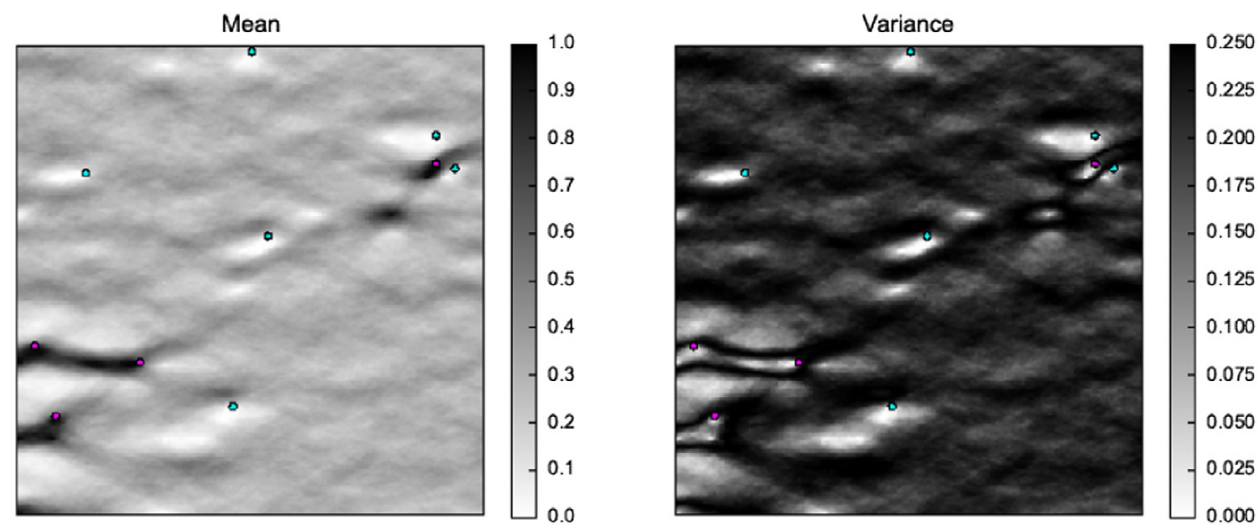

(c) $G=8$.

Fig. 1. The mean and variance of 100 simulations using 1,4 , and 8 multiple grids, respectively. The training image and data template used are shown in Figs. 4(a) and 2(c), respectively. Purple circles represent hard data points labeled ' 1 ' and blue circles represent the hard data points labeled ' 0 '. (For interpretation of the references to colour in this figure legend, the reader is referred to the web version of this article.) 


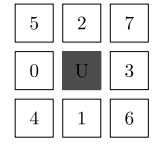

(a) Square data template whose size is 8 .

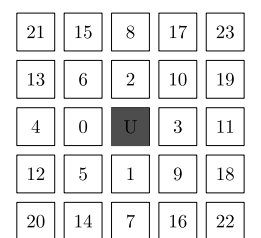

(b) Square data template whose size is 24 .

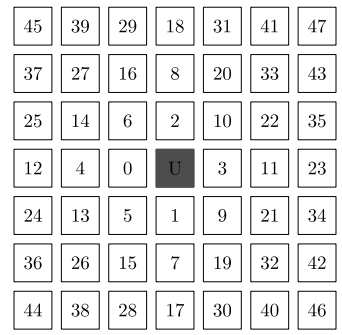

(c) Square data template whose size is 48 .

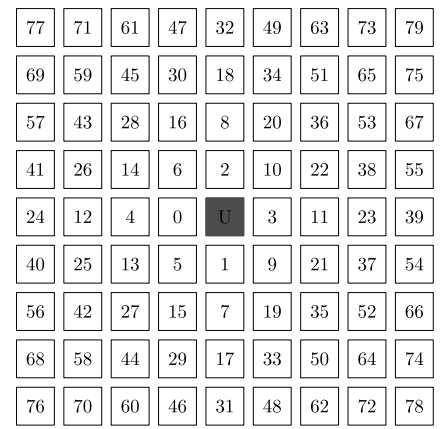

(d) Square data template whose size is 80 .

\begin{tabular}{|l|l|l|l|l|l|l|l|l|l|l|l|}
\hline 51 & 45 & 36 & 26 & 16 & 8 & 18 & 28 & 38 & 47 & 53 \\
\hline 43 & 34 & 24 & 14 & 6 & 2 & 10 & 20 & 30 & 40 & 49 \\
\hline 32 & 22 & 12 & 4 & 0 & $\mathrm{U}$ & 3 & 11 & 21 & 31 & 41 \\
\hline 42 & 33 & 23 & 13 & 5 & 1 & 9 & 19 & 29 & 39 & 48 \\
\hline 50 & 44 & 35 & 25 & 15 & 7 & 17 & 27 & 37 & 46 & 52 \\
\hline
\end{tabular}

(e) East-west elongated data template.

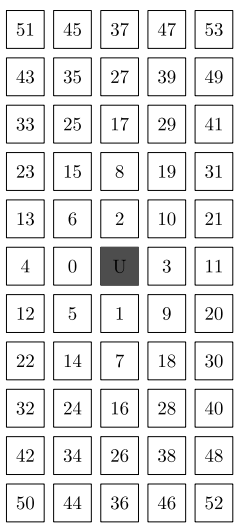

(f) North-south elongated data template.

Fig. 2. All templates used in the experiments.

Accordingly, it is important to determine the appropriate number of multiple grids in a quantitative manner.

For certain MPS methods, we refer to the simulation scale as the coarsest scale, i.e., the furthest grid that the simulation can model, and the target scale as the coarsest scale that results in a statistically significant and sufficiently large spatial association. Honarkhah and Caers (2010) have proposed using the maximum second derivative of the log-likelihood of the mean entropy of different templates to find the optimal template size. From another perspective, the correlation between surface objects at different distances can also be used to determine if there is large-scale structural information at a certain scale. Join Count Statistics (JCSs) (Cliff and Ord, 1970, 1981) are commonly used to detect the existence of such patterns. JCSs are also closely related to the concept of connected components 
(Hovadik and Larue, 2007), which are known to be important in describing reservoirs (Renard and Allard, 2013). It is instructive to determine the scale that should be considered and the number of multiple grids using JCSs.

This paper proposes a quantitative method for estimating the minimal number of multiple grids that can produce simulations of acceptable quality. The method uses the degree of spatial association between objects to estimate the coarsest scale that the simulation should consider, i.e., the target scale. In doing so, the spatial association is quantified using a modified JCS. The minimal number of multiple grids is estimated by setting the coarsest scale that the simulation can consider (i.e., the simulation scale) to be no less than the target scale. A series of experiments are performed to validate the new method. Our results suggest that the new method can determine the appropriate number of multiple grids for simulations. The results also demonstrate that the new method is not sensitive to the size and geometry of the data template, or to the geometric structures of the target surface objects. This issue was primarily discussed in Bai and Ge (in press), which gave a brief introduction of the method and a very limited example. This paper gives the theoretical description about the proposed method and offers a solution to automatically adapt to the grid size and the template configuration. Furthermore, real-life experiments are used to consolidate the proposed method.

The remaining part of this paper proceeds as follows. Section 2 contains a review of some basic concepts and the general procedure of the multiple grid-based SNESIM algorithm. Section 3 introduces the proposed method for estimating the minimal number of multiple grids by tuning the simulation scale using the target scale. Section 4 describes a series of two-dimensional experiments and one three-dimensional experiment. These are used to validate the new method for isotropic and anisotropic target surface objects. This section also includes a sensitivity analysis regarding the data template size and geometry. Section 5 discusses the influence of the geometric structure of the target surface objects, the training image size, and the data template configuration on the proposed method. It also examines the relationship between insufficient replicas of large data events and the proposed method. Section 6 concludes the paper.

\section{Preliminaries of multiple grid-based SNESIM}

A detailed description of multiple point simulation and the SNESIM algorithm can be found in Strebelle (2000). This section briefly introduces the preliminaries of the SNESIM algorithm. The first step of SNESIM is to construct a two- or three-dimensional regular Cartesian grid (for simplicity, a regular grid $g$ ) with hard data assigned to the closest grid cells. The regular grid $g$ is a tessellation of two-dimensional (or three-dimensional) Euclidean space using unit squares (or cubes). Each unit square (or cube) is called a cell of $g$. Cell $i$ of $g$ is represented using the smallest two (or three) coordinates $\{x(i), y(i)\}$ (or $\{x(i), y(i), z(i)\}$ ) of all the vertices in the unit square (or cube). Any cell whose coordinates are integer multiples of $2^{i}$ is called a $2^{i}$ th cell of $g$.

The training image and the data template are two indispensable parameters of SNESIM. A training image can be a regular grid with the same granularity as the constructed regular grid $g$. This can be a classification result (Boucher, 2008) from a remotely sensed image, an image sketched by a specialist from related fields, or any images with the structural characteristics of the target surface objects (Strebelle, 2000). The data template defines the neighbors of a cell. The concept of the data template and the data event are defined as follows.

Definition 1. A data template is a set of $n$ vectors $\tau_{n}=\left\{\mathbf{h}_{i}, i=1, \ldots, n\right\}$, where $\mathbf{h}_{i}$ contains the coordinates of the $i$ th vector of the data template, and at least one coordinate of $\mathbf{h}_{i}$ is not equal to zero. Any $n^{\prime} \leq n$ vectors in $\tau_{n}$ constitute a sub data template of $\tau_{n}$.

Definition 2. A data event $d_{n}$ of a cell in a regular grid $g$ is a pair $\left(\tau_{n}, \sigma_{n}\right)$, where $\sigma_{n}=\left\{v_{i}, i=1, \ldots, n\right\}$ contains the $n$ values associated with the $n$ vectors $\left\{\mathbf{h}_{i}, i=1, \ldots, n\right\}$ of $\tau_{n}$.

After preparing the training images and the data template, the structural patterns of the target facies are extracted from the training images and saved in a search tree. During the simulation, the data event for each unsampled cell is constructed using hard data and previously simulated cells within 
the data template. The data event is then used to extract a probability model from the search tree, and the value of the current cell is randomly drawn from the derived probability model. Details of the data structure and operation of a search tree can be found in Strebelle (2000).

Strebelle (2000) proposed using multiple-grid simulations for reproducing large-scale structures in the training image using a relatively small predefined template. A typical multiple-grid SNESIM decomposes the simulation process into several sub-simulations on different sub-grids. The $g$ th subsimulation from the bottom is performed on the $g$ th grid of the regular grid $g$.

Definition 3. The $g$ th grid of a regular grid $g$ is constituted by every $2^{g-1}$ th cell of $g$. If $g=1$, then the $g$ th grid is called the finest grid of $g_{\text {. If }} g_{1}>g_{2}$, then the $g_{1}$ th grid is coarser than the $g_{2}$ th grid and the $g_{2}$ th grid is finer than the $g_{1}$ th grid.

There are a total of $G$ sub-grids in the multiple-grid simulation, ranging from the Gth grid to the finest grid. Thus, $G$ is the number of multiple grids. All sub-simulations are performed consecutively from the $G$ th grid to the finest grid. For the $g$ th $(1 \leq g \leq G)$ grid, SNESIM rescales the predefined data template $2^{g-1}$ times in all directions. That is, each vector in the data template is enlarged $2^{g-1}$ times. The search tree for the current grid is constructed using the enlarged data template, so as to reproduce large-scale structural information. Each unsampled cell of the current grid is simulated using the search tree and the enlarged data template. The simulated cells in the current grid are used as hard data for the finer grids. Details of the multiple-grid-based SNESIM can be found in Strebelle (2000).

\section{Method}

As noted by Strebelle (2000) and Liu (2006), if the number of multiple grids is too small, there will be a lack of consideration of large-scale structural information, eventually leading to a poor reproduction of target surface objects. Accordingly, the number of multiple grids should be set in terms of the criterion that the simulation scale should be larger than the target scale. Here, the scale mainly refers to the spatial lag (Dungan et al., 2002), i.e., the distances between cells. The spatial characteristics may change at different scales. Spatial association is an effective approach to analyze the spatial scale, i.e., the number of multiple grids that should be used.

Our method mainly comprises three steps. First, the simulation scale can be inferred in terms of the data template and the number of multiple grids. Second, the scale up to which the target surface objects have significant structural characteristics can be used as the target scale. JCSs (Cliff and Ord, 1970,1981 ) can be used to determine this coarsest scale. Finally, the number of multiple grids that ensures the simulation scale is no less than the target scale used as an estimate of the minimal number of multiple grids.

\subsection{The simulation scale}

The predefined data template $\tau_{n}$ limits the most distant cell that can be taken into account during the simulation of the current cell. To account for the anisotropic structural information of target surface objects, the coarsest scale that can be considered is calculated for each axis.

Let $S_{1}^{m}$ represents the maximum absolute coordinate on the $m$ axis. Then, $S_{1}^{m}$ is the coarsest scale that can be considered on the $m$-axis of the finest grid. The subscript ' 1 ' represents the finest grid. The coarsest scale that can be considered for all axes on the finest grid of the two-dimensional (or threedimensional) data template is a vector $\mathbf{S}_{1}=\left(S_{1}^{x}, S_{1}^{y}\right)\left(\right.$ or $\left.\mathbf{S}_{1}=\left(S_{1}^{x}, S_{1}^{y}, S_{1}^{z}\right)\right)$. Consider the data template in Fig. 2(c) as an example. For the $x$ - and $y$-axes, $S_{1}^{x}=S_{1}^{y}=3$. Finally, $\mathbf{S}_{1}=(3,3)$.

For the $g$ th grid, because the predefined data template $\tau_{n}$ is re-scaled, the absolute coordinates in all directions are also enlarged $2^{g-1}$ times. Accordingly, the simulation scale on the $m$-axis of the $g$ th grid is $S_{g}^{m}=2^{g-1} S_{1}^{m}$. If the number of multiple grids being used is $G$, then the simulation scale on the $m$-axis is $S_{G}^{m}$. For the data template in Fig. $2(\mathrm{c}), S_{G}^{x}=S_{G}^{y}=24$ if $G=4$. 


\subsection{The target scale}

The prior structural information of surface objects is stored in the training images, so it is reasonable to infer the target scale from the training images. However, several training images may be used to simulate different simulation grids (Liu, 2006). In such situations, the target scale is estimated from the training image that contains the largest-scale structural information. This is because other training images are used for finer grids and may only carry local structural information of the target surface objects.

The correlation between surface objects at a certain distance can be used to determine whether there is large-scale structural information at a certain scale. If objects have statistically significant structural information at a certain scale, then there will be certain patterns in the co-occurrences of certain categories at these scales. JCSs are commonly used to detect the existence of such patterns.

JCSs use the joins between cells to test for spatial associations. Joins can be defined by an adjacency matrix $A=\left[a_{i j}\right]_{n \times n}$, where $n$ is the number of cells and $i, j$ represent two cells in a grid. $a_{i j}=1$ if cells $i$ and $j$ are 1 -adjacent; otherwise, $a_{i j}=0$. The 1-adjacency of two cells can be established using any connectivity algorithm (Dale and Fortin, 2014). The $k$ th order adjacency matrix $A^{k}$ can be used to measure high order spatial associations. It represents the relations between cells that are not 1adjacent. $A^{k}$ can be recursively derived using the concept of relation composition or just using the distance between cells (Bai et al., 2016) in terms of the requirements of applications.

In our model, the spatial associations are measured on all axes at different scales. For the $m$ th axis, the $k$ th order adjacency matrix for scale $k$ is defined as $A_{m}^{k}=\left[a_{i j}\right]_{n \times n}$, where

$$
a_{i j}= \begin{cases}1, & |m(i)-m(j)|=k \wedge \forall m^{\prime} \neq m, m^{\prime}(i)=m^{\prime}(j) \\ 0, & \text { otherwise, }\end{cases}
$$

where $m(i)$ corresponds to the coordinate of the cell $i$ along the $m$ th axis.

If $a_{i j}=1$, then there is a join between cells $i$ and $j$. If a join connects two cells with different categories, then the join is called a diff-join (i.e. rs-join (Cliff and Ord, 1981)). $N_{\text {diff }}$ denotes the number of all diff-joins. $N_{\text {all }}$ denotes the number of all joins.

JCSs compare $N_{\text {diff }}$ with the theoretically $E\left(N_{\text {diff }}\right)$ expected number from a completely random spatial distribution, i.e., $J C S=N_{\text {diff }}-E\left(N_{\text {diff }}\right)$. If $J C S<0$, then cells from the same category tend to agglomerate in space (positive spatial association). If $J C S>0$, then cells from different categories tend to agglomerate (negative spatial association) (Bai et al., 2016).

As an index for significance testing, JCS is a relative quantity associated with the observed and expected join number. As well as significance testing, it is necessary to measure the degree of spatial association when estimating the target scale (Bai et al., 2016; Leslie and Kronenfeld, 2011). If the degree of spatial association is too small, the structural information at this scale can be ignored. This means the computation time can be reduced during the simulation without significantly affecting the simulation quality.

In this work, the JCS was modified to better reflect two extreme situations: completely positive and completely negative spatial associations (Bai et al., 2016). $P($ diff $)=N_{\text {diff }} / N_{\text {all }}$ is the probability of observing diff-joins, and $P_{E}($ diff $)=E\left(N_{\text {diff }}\right) / N_{\text {all }}$ is the expected probability of observing diff-joins under the assumption of a random distribution. Let $S=P_{E}($ diff $)-P($ diff $)$, which is opposite in sign to the JCS. This can be normalized to the range $[-1,1]$ using

$$
\mathrm{NJCS}=\left\{\begin{array}{l}
\frac{S}{1-P_{E}(\text { diff })}, \quad S \leq 0 \\
\frac{S}{P_{E}(\text { diff })}, \quad S>0 .
\end{array}\right.
$$

A larger NJCS corresponds to a smaller value of $P($ diff $)$. In other words, a smaller probability of observing diff-joins means that it is more likely that cells from the same category congregate. When NJCS equals $1, P($ diff $)=0$. This means that any join in the spatial data must connect two cells of the same category-a completely positive spatial association. Conversely, a smaller NJCS corresponds to a larger value of $P$ (diff). That is, a larger probability of observing diff-joins means that it is more 
likely that cells of different categories will congregate. When NJCS equals $-1, P($ diff $)=1$. This means that any join in the spatial data must connect two cells of different categories-a completely negative spatial association.

As JCS is not confined to two categories, NJCS can be effectively used to measure the degree of spatial association. Accordingly, the proposed method is also appropriate for multiple category scenarios. Suppose that there are five categories denoted as $C_{1}, C_{2}, C_{3}, C_{4}, C_{5}$, respectively. We can estimate the marginal probability of different categories through the proportion of every categories to all categories. Then $P_{E}$ (diff $)=\sum_{i=1}^{5} \sum_{j \neq i, j=1}^{5} P\left(C_{i}\right) P\left(C_{j}\right)$ is calculated under the assumption of completely random spatial distribution. Meanwhile, $N_{\text {diff }}$ can also be obtained through counting the joins that connect two cells with different category. Next we compute $P_{\text {diff }}=N_{\text {diff }} / N_{\text {all }}$. Finally, it is easy to calculate NJCS using Eq. (1). The detailed procedure of calculating the $k$ th order NJCS for multiple categories is shown in Algorithm 1.

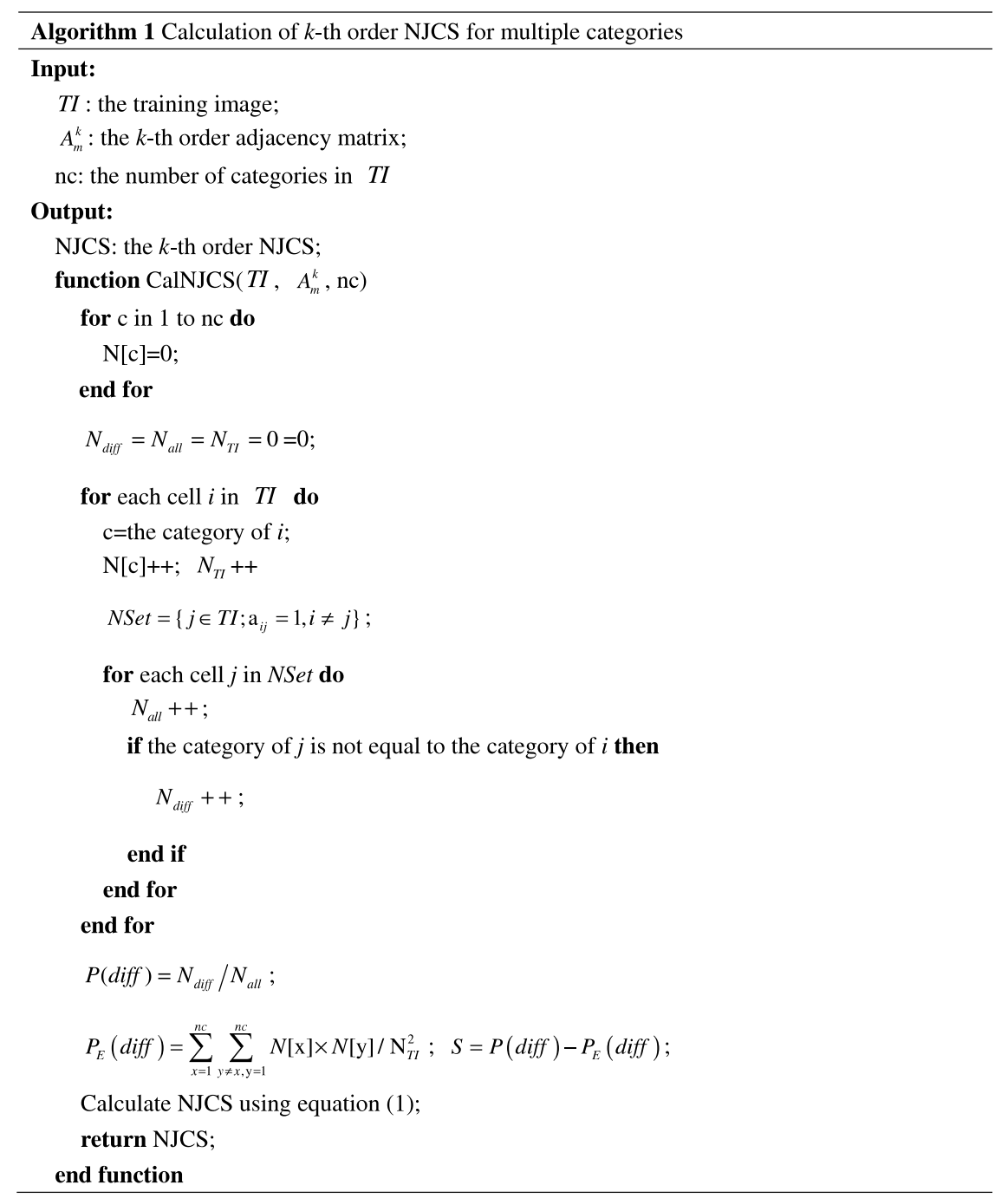

When the absolute value of NJCS is very small (e.g., less than 0.1 ), it is reasonable to assume that there is no significant large-scale structural information. For example, suppose that there are two categories $\mathrm{C} 1$ and $\mathrm{C} 2$. If $P(C 1)=0.3$ and $P(C 2)=0.7$, then $P_{E}($ diff $)=P(C 1) P(C 2)=0.21$. If 
NJCS $=0.1$, then $P_{E}($ diff $)-P($ diff $)=0.021$. This means that the difference between the observed value and the expected value from the random distribution is very small. Thus, there will be little structural information remaining at this scale. The consideration of structural information at this scale cannot significantly improve the simulation quality.

Generally, NJCS decreases as $k$ increases. When the JCS is not statistically significant or NJCS is smaller than the given threshold, the corresponding scale $k$ can be set as the target scale on this axis, i.e., $T^{m}=k$. The target scales on all axes for the two-dimensional (or three-dimensional) training image constitute a vector $\mathbf{T}=\left(T^{x}, T^{y}\right)\left(\right.$ or $\left.\mathbf{T}=\left(T^{x}, T^{y}, T^{z}\right)\right)$.

\subsection{Finding the suitable minimal number of multiple grids for simulation}

After calculating the coarsest scale $S^{m}$ that can be taken into account by the data template for the finest grid and the target scale of the target surface objects $T^{m}$, the minimal number of multiple grids can be estimated in a straightforward manner. On the $m$-axis, the minimal number of multiple grids can be estimated based on the criterion that the simulation scale should be no less than the target scale. Formally,

$$
G^{m}=\left\lceil\log _{2}\left(T^{m} / S_{1}^{m}\right)\right\rceil+1 .
$$

To account for the largest-scale structural information on different axes, the minimal number of multiple grids is estimated using $G_{E}=\max \left(\left\{G^{m}, m \in\{x, y\}(m \in\{x, y, z\})\right\}\right)$.

The proposed method is applicable with training images having more than two categories. The calculation of $S^{m}$ and $T^{m}$ only depends on the data template and not the number of categories in the training image. Additionally, no matter how many categories are present in the training image, NJCS can take all of them into account simultaneously. When there are $N$ classes, Eq. (1) needs to calculate $P$ (diff) and $P_{E}$ (diff). These two probabilities do not need to be calculated for each category. They are calculated for all categories once and for all. Meanwhile, for the cases that one particular category has much greater range of continuity than all the other categories, the NJCS can also estimate the coarsest scale for each category through merging all other categories into one category.

\section{Experiment design}

A series of experiments were designed to validate the effectiveness of the proposed method. The simulated surface objects were of three different geometrical structures: curvilinear surface objects, disc-shaped surface objects, and ellipse-shaped surface objects. The training image for the curvilinear surface objects was taken from Strebelle (2000). The proposed method was first applied to twodimensional simulations using different training images and different data templates, to investigate the method's robustness when estimating the minimal number of multiple grids. Second, a threedimensional simulation example is presented to show that the proposed method can select an appropriate multiple-grid number for a three-dimensional simulation.

All simulations considered two categories dependent on the colors in the training image: Black and White. Hard data points can lead to conflicts between hard data points and training images, which may result in a poor reproduction of certain structures in the training images (Strebelle, 2000). One solution is to perform an unconditional simulation from which to extract conditioning data. This ensures that the conditioning data are compatible with the training image. The maximum number of conditioning data was set to 32 , the minimum number of replicas (mincd) was set to 20 , and the marginal probabilities of the black and white classes were set to 0.3 and 0.7 , respectively. The simulation size was set to $200 \times 200$ for the $2 \mathrm{D}$ case and $100 \times 100 \times 100$ for the 3D case. The training images were rescaled to test the effectiveness of the proposed method. For example, the training image for the disc-shaped surface objects was rescaled to $0.5,1,1.5,1.75$, and 2 times its original size. Experiments for the curvilinear objects were also performed using five different scales. Similar trends could be found in the experimental results. For simplicity and without loss of generality, only three scales for the curvilinear objects were used, i.e. the training images containing curvilinear surface objects were rescaled to $0.5,1$ and 2 times their original size. 
Table 1

The target scales of different rescaled training images and the corresponding estimated minimal numbers of multiple grids in terms of the magnification factor and data template used.

\begin{tabular}{rlllll}
\hline ROW ID & Shape & Data template used & Magnification factor & $T$ & $(7,7)$ \\
\hline 1 & Disc & Fig. 2(c) & 0.50 & $(13,14)$ & 3 \\
2 & Disc & Fig. 2(c) & 1.00 & $(20,21)$ & 4 \\
3 & Disc & Fig. 2(c) & 1.50 & $(24,25)$ & 5 \\
4 & Disc & Fig. 2(c) & 1.75 & $(27,28)$ & 5 \\
5 & Disc & Fig. 2(c) & 2.00 & $(19,5)$ & 6 \\
6 & Curvilinear & Fig. 2(a) & 1.00 & $(19,5)$ & 5 \\
7 & Curvilinear & Fig. 2(b) & 1.00 & $(9,3)$ & 3 \\
8 & Curvilinear & Fig. 2(c) & 0.50 & $(19,5)$ & 4 \\
9 & Curvilinear & Fig. 2(c) & 1.00 & $(39,11)$ & 5 \\
10 & Curvilinear & Fig. 2(c) & 2.00 & $(19,5)$ & 4 \\
11 & Curvilinear & Fig. 2(d) & 1.00 & $(19,5)$ & 3 \\
12 & Curvilinear & Fig. 2(e) & 1.00 & $(19,5)$ & 4 \\
13 & Curvilinear & Fig. 2(f) & 1.00 & $(15,7)$ & 5 \\
14 & Ellipse & Fig. 2(a) & 1.00 & $(15,7)$ & 4 \\
15 & Ellipse & Fig. 2(b) & 1.00 & $(15,7)$ \\
16 & Ellipse & Fig. 2(c) & 1.00 & 1.00 & 3 \\
17 & Ellipse & Fig. 2(d) & & \\
\hline
\end{tabular}

All experiments followed the same procedure: first estimate the minimal number of multiple grids using the proposed method, then perform simulations using number of multiple grids from 1 to 6 . All simulations were run 100 times, but our results display only one realization. As discussed in Section 3.2, there will be little structural information remaining at this scale when the marginal probability of two categories is 0.3 and 0.7 and the NJCS is less than 0.1 . Accordingly, the NJCS threshold for all experiments was set to 0.1 . The sub-figures surrounded by black boxes in all simulations are the simulation results given by the estimated minimal number of multiple grids.

\subsection{Two-dimensional simulations}

\subsubsection{Simulation using different training images}

Two different training images were used to validate the effectiveness of the proposed method. The training images for the disc-shaped and curvilinear surface objects were $400 \times 300$ and $250 \times 250$ cells, respectively, in size. Given the template used, $S_{1}=(3,3)$ (see Fig. 2(c)).

The new method was used to determine the optimal number of multiple grids for each rescaled training image of disc-shaped surface objects. For example, $T=(13,14)$ for the original training image with 0.1 as the NJCS threshold. Because $S_{1}=(3,3)$, Eq. (2) implies that the estimated minimal number of multiple grids was four for both axes. Rows 1-5 in Table 1 summarize the target scale and the estimated minimal number of multiple grids for the rescaled training images. For each rescaled training image, six groups of simulations were run using multiple grid numbers from 1 to 6 . The first simulation results for the six groups of rescaled training images are shown in Fig. 3. The first row of Fig. 3 contains the original training image of the disc-shaped surface objects, and the other rows show the same rescaled training image at $0.5-2$ times its original size. From left to right, each column shares the same number of multiple grids (from 1 to 6 ). For example, Fig. 3(d3) is a simulation using $G=3$ and a training image that is 1.5 times the size of the original training image.

The diameter of each disc in the original training image is 20 . It is much larger than the scale estimated for the training images. The target scale of the training image not only reflects the shape of the surface objects, but also reflects the arrangement of objects. Although the disc-shaped objects are isotropic, the distribution of the surface objects on different axes may differ a little. Accordingly, it is logical that slight anisotropic disc-shaped objects are present in the simulations. For the same reason, the dimension of individual objects is not sufficient for predicting how many multiple grids should be used.

Rows 8-10 of Table 1 summarize the target scales and the estimated number of multiple grids for all rescaled training images of curvilinear surface objects. The first simulations for the curvilinear 


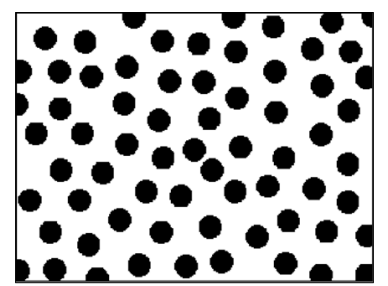

(a) Training image containing the disc-shaped objects

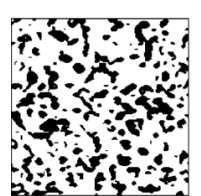

(b1) $\mathrm{G}=1$

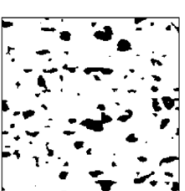

(c1) $\mathrm{G}=1$

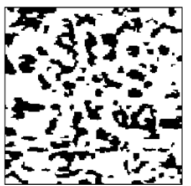

(d1) $\mathrm{G}=1$

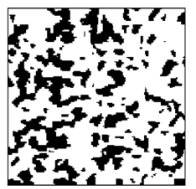

(e1) $\mathrm{G}=1$

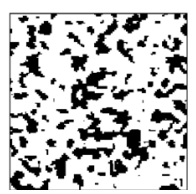

(f1) $\mathrm{G}=1$

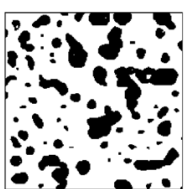

(b2) $\mathrm{G}=2$

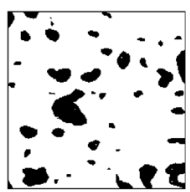

(c2) $\mathrm{G}=2$

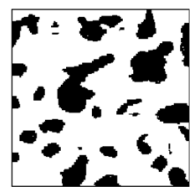

(d2) $\mathrm{G}=2$

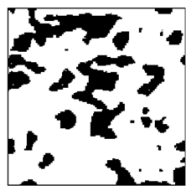

(e2) $\mathrm{G}=2$

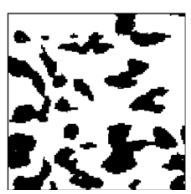

(f2) $\mathrm{G}=2$

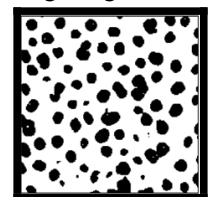

(b3) $\mathrm{G}=3$

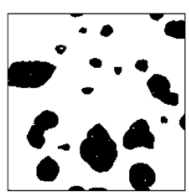

(c3) $\mathrm{G}=3$

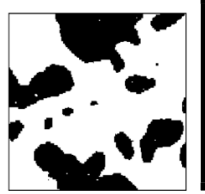

(d3) $\mathrm{G}=3$

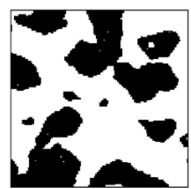

(e3) $\mathrm{G}=3$

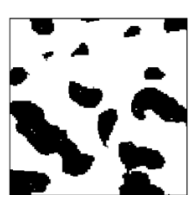

(f3) $\mathrm{G}=3$

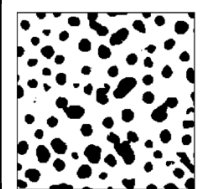

(b4) $\mathrm{G}=4$

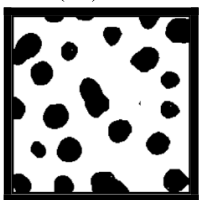

(c4) $\mathrm{G}=4$

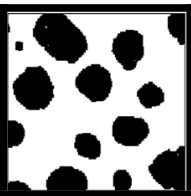

(d4) $\mathrm{G}=4$

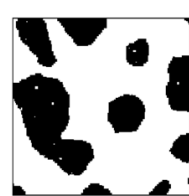

(e4) $\mathrm{G}=4$

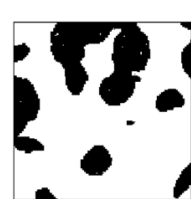

(f4) $\mathrm{G}=4$

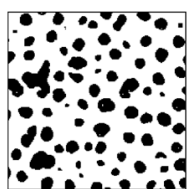

(b5) $\mathrm{G}=5$

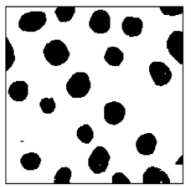

(c5) $\mathrm{G}=5$

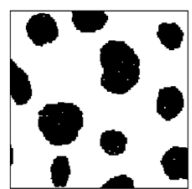

(d5) $\mathrm{G}=5$

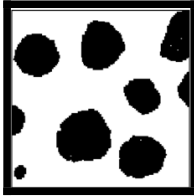

(e5) $\mathrm{G}=5$

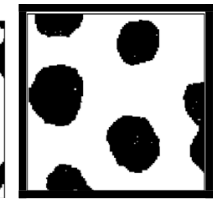

(f5) $\mathrm{G}=5$

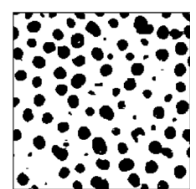

(b6) $\mathrm{G}=6$

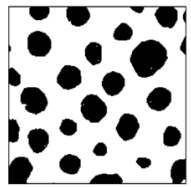

(c6) $\mathrm{G}=6$

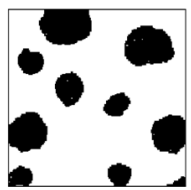

(d6) $\mathrm{G}=6$

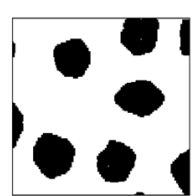

(e6) $\mathrm{G}=6$

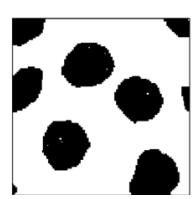

(f6) $\mathrm{G}=6$

Fig. 3. Training image and simulation results of the disc-shaped surface objects. The second to the last rows contain the simulation results using rescaled training images at $0.5,1,1.5,1.75$ and 2 times the original size, respectively. The subfigures' captions show the number of multiple grids used. The figures highlighted in bold represent the optimal number of multiple-grids.

surface objects are shown in Fig. 4. The first row of the figure contains the original training image. Rows 2-4 show the same rescaled training image from 0.5 to 2 times its original size, and each column corresponds to the same number of multiple grids (1-6 from left to right).

\subsubsection{Simulation using different templates}

East-west and north-south elongated data templates, and data templates of different sizes, were used to investigate how the data template geometry and size influence the estimated minimal number of multiple grids. The east-west and north-south elongated data templates are shown in Fig. 2(e), (f). 


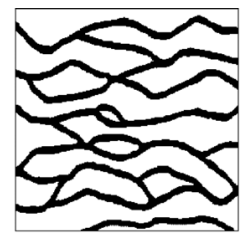

(a) Training image containing the curvilinear surface objects.

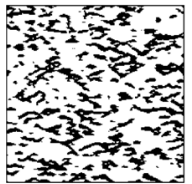

(b1) $\mathrm{G}=1$

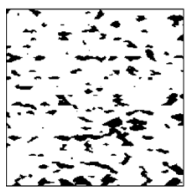

(c1) $\mathrm{G}=1$

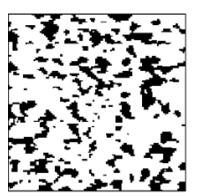

(d1) $\mathrm{G}=1$

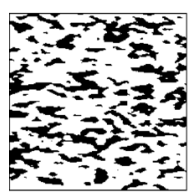

(e1) $\mathrm{G}=1$

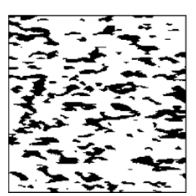

(f1) $\mathrm{G}=1$

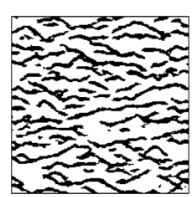

(b2) $\mathrm{G}=2$

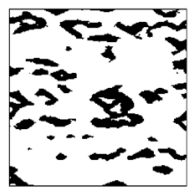

(c2) $\mathrm{G}=2$

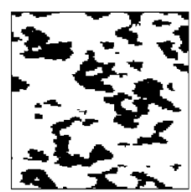

(d2) $\mathrm{G}=2$

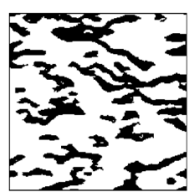

(e2) $\mathrm{G}=2$

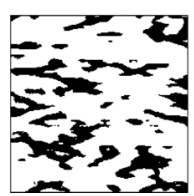

(f2) $\mathrm{G}=2$

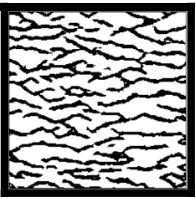

(b3) $\mathrm{G}=3$

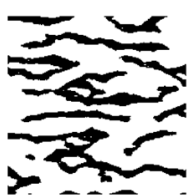

(c3) $\mathrm{G}=3$

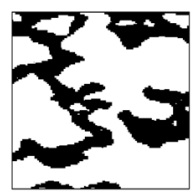

(d3) $\mathrm{G}=3$

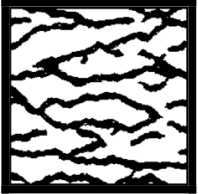

(e3) $\mathrm{G}=3$

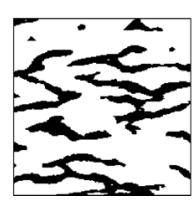

(f3) $\mathrm{G}=3$

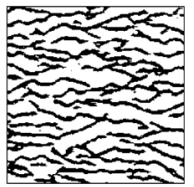

(b4) $\mathrm{G}=4$

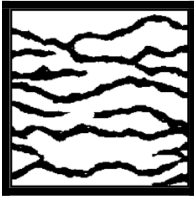

(c4) $\mathrm{G}=4$

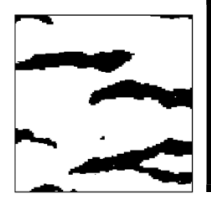

(d4) $\mathrm{G}=4$

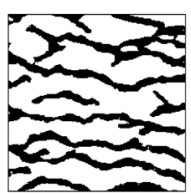

(e4) $\mathrm{G}=4$

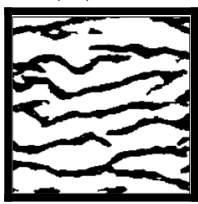

(f4) $\mathrm{G}=4$

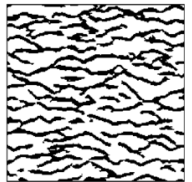

(b5) $\mathrm{G}=5$

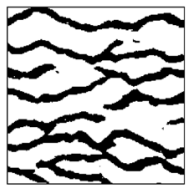

(c5) $\mathrm{G}=5$

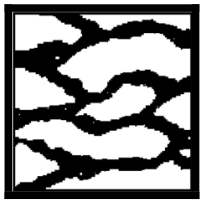

(d5) $\mathrm{G}=5$

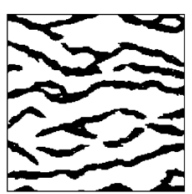

(e5) $\mathrm{G}=5$

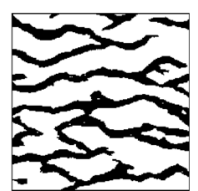

(f5) $\mathrm{G}=5$

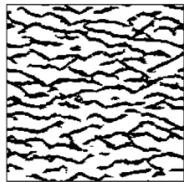

(b6) $\mathrm{G}=6$

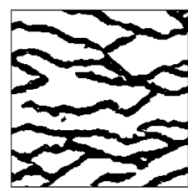

(c6) $\mathrm{G}=6$

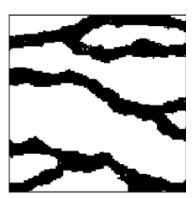

(d6) $\mathrm{G}=6$

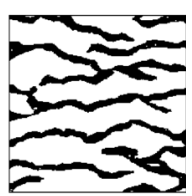

(e6) $\mathrm{G}=6$

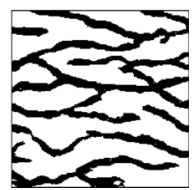

(f6) $\mathrm{G}=6$

Fig. 4. The training image and simulation results of the curvilinear surface objects. The second to fourth rows contain the simulation results using rescaled training images at $0.5,1$, and 2 times the original size, respectively. The fifth row contains the simulation results using the original training image and the east-west elongated template. The last row contains the simulation results using the original training image and the north-south elongated template. The subfigures' captions show the number of multiple grids used. The figures highlighted in bold represent the optimal number of multiple-grids.

$S_{1}=(5,2)$ for the east-west elongated template, and $S_{1}=(2,5)$ for the north-south elongated template. The original training images containing the curvilinear surface objects were used in this experiment. Rows 12-13 of Table 1 summarize the target scales and the estimated number of multiple grids for the elongated data template simulations. The first simulation results using the east-west and north-south elongated data templates and different numbers of multiple grids are shown in Fig. 4(e1)-(f6). Fig. 4(e1)-(e6) show the results using the east-west elongated data templates, and Fig. 4(f1)-(f6) show the results using the north-south elongated data templates. From left to right, each column in the last two rows of Fig. 4 shares the same number of grids (from 1 to 6 ). 
The influence of the data template size on the estimates of the minimal number of multiple grids was analyzed using two experiments. Three data templates of different sizes were investigated. Fig. 2(a)-(d) show the data templates, all of which were square. The sizes of the four data templates were $8,24,48$, and 80 respectively, and the corresponding $S_{1}$ values were $(1,1),(2,2),(3,3)$ and $(4,4)$. The ellipse-shaped and curvilinear surface objects were used as examples. The size of the training images containing these two types of surface objects was $400 \times 300$ and $250 \times 250$, respectively. The number of multiple grids estimated using different data templates is listed in Table 1. Simulations for the curvilinear surface objects and ellipse-shaped surface objects using data templates of different sizes and different numbers of grids are shown in Figs. 5 and 6 . In these two figures, (b1)-(b8) contain simulations using 1-8 multiple grids and the data template shown in Fig. 2(a); (c1)-(c6) show simulations using 1-6 multiple grids and the data template shown in Fig. 2(b); (d1)-(d6) are the results using 1-6 multiple grids and the data template shown in Fig. 2(c); and (e1)-(e6) show the simulation results using 1-6 multiple grids and the data template shown in Fig. 2(d). The first rows of these two figures are the training images used in the simulations.

\subsection{Three-dimensional simulations}

A three-dimensional experiment was conducted to demonstrate that the proposed method is appropriate for three-dimensional simulations. Because three-dimensional training volumes are hard to obtain for practical applications (Strebelle, 2000), many MPS algorithms generate threedimensional simulations using two-dimensional training images (Comunian et al., 2012; Strebelle, 2000). In this study, we generated three-dimensional simulation results using the technique proposed by Strebelle (2000). This method generates two simulations for each unsampled node using either $\mathrm{XY}$-oriented or YZ-oriented training images. The two simulations are merged through a combination algorithm proposed by Journal (2000).

The data templates used for the XY- and YZ-oriented simulations had 48 neighbors (Fig. 2(c)). The other parameters for the three-dimensional simulations were the same as for the two-dimensional simulations. Fig. 7(a) and (b) are the XY- and YZ-oriented training images, respectively. These two training images were taken from Strebelle (2000). Fig. 7(c1)-(c6) show one realization for 1-6 multiple grids. The sub-figure surrounded by a black box is the simulation result given by the estimated minimal number of multiple grids. Fig. 7(d1)-(d6) are sections cut from three dimensional results shown in Fig. 7(c1)-(c6), respectively.

Because two training images were used for the three-dimensional simulations, the minimal numbers of multiple grids were estimated for both training images, and the largest was used. The estimated minimal number of multiple grids was four for the XY-oriented training image and two for the YZ-oriented training images. Accordingly, the estimated minimal number of multiple grids was four.

\section{Discussion}

The simulation results from all experiments share some common characteristics. For a small number of grids, it is hard to discern the target surface objects. That is, the simulation results give a poor reproduction of the structure of the training images. As the number of multiple grids increases, the geometric structures of the target surface objects become gradually more pronounced, because an increasing amount of large-scale spatial structural information is taken into account. When the number of multiple grids is greater than the minimal multiple grid number estimated using the proposed method, the simulation quality does not significantly improve. This trend is also observed in the three-dimensional simulations.

Fig. 8 shows the mean vertical and horizontal variogram charts of the simulation results using the original training image containing curvilinear surface objects and the data template of size 48, east-west elongated template, and the north-south elongated data template. It can be seen that, when the number of multiple grids is greater than the estimated value, the mean variogram of 100 simulations does not change significantly. Take the vertical variogram chart in Fig. 8(c) as an example. 


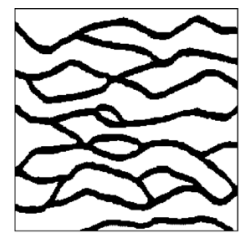

(a) Training image containing the curvilinear surface objects.

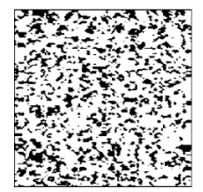

(b1) $\mathrm{G}=1$

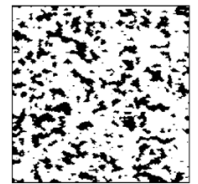

(b2) $\mathrm{G}=2$

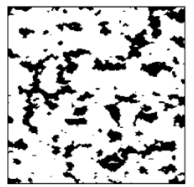

(b3) $\mathrm{G}=3$

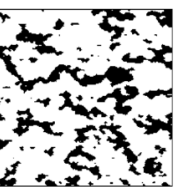

(b7) $\mathrm{G}=7$

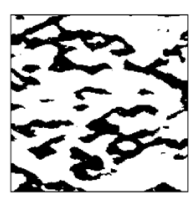

(c3) $\mathrm{G}=3$

(c2) $\mathrm{G}=2$

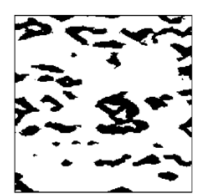

(d2) $\mathrm{G}=2$

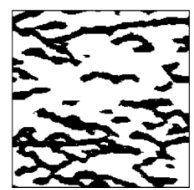

(e2) $\mathrm{G}=2$

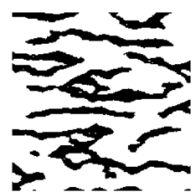

(d3) $\mathrm{G}=3$

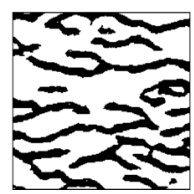

(e3) $G=3$

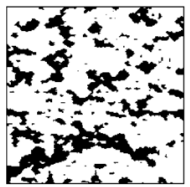

(b4) $\mathrm{G}=4$

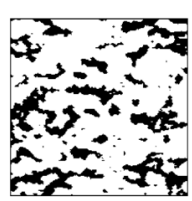

(b5) $\mathrm{G}=5$

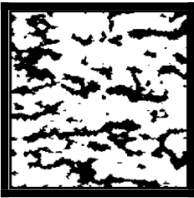

(b6) $\mathrm{G}=6$

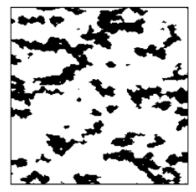

(b8) $\mathrm{G}=8$

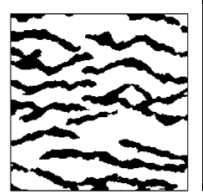

(c4) $\mathrm{G}=4$

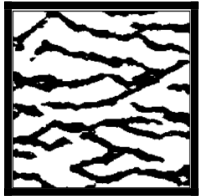

(c5) $\mathrm{G}=5$

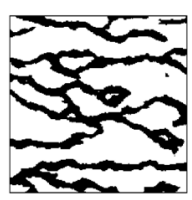

(c6) $\mathrm{G}=6$

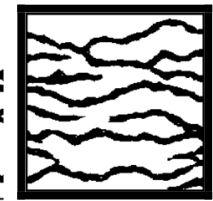

(d4) $\mathrm{G}=4$

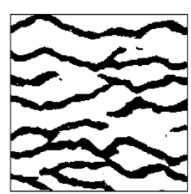

(d5) $\mathrm{G}=5$

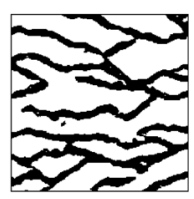

(d6) $\mathrm{G}=6$

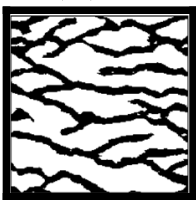

(e4) $\mathrm{G}=4$

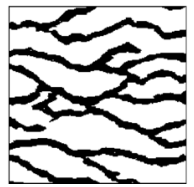

(e5) $\mathrm{G}=5$

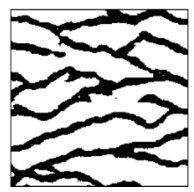

(e6) $\mathrm{G}=6$

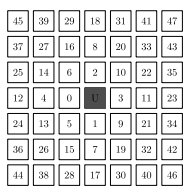

(f3) template for $d 1$ to $d 6$

(f4) template for e1 to e6

(f1) template for b1 to b8

(f2) template for $\mathrm{c} 1$ to $\mathrm{c} 6$

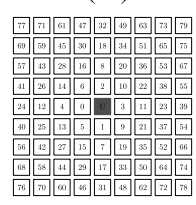

Fig. 5. The training image and simulation results of the curvilinear surface objects using data templates with different sizes. The subfigures' captions for (b1)-(e6) are the number of multiple grids used. The subfigures' captions for (f1)-(f4) show the data template used by simulations. The figures highlighted in bold represent the optimal number of multiple-grids.

With fewer than four grids, the mean variograms at lags of 10-25 are significantly smaller than for the original training image. This means that the statistical characteristics of the structure given by the simulation are different from those of the original training image. 


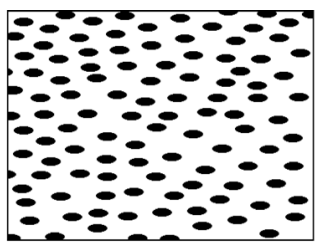

(a) Training image containing the curvilinear surface objects.

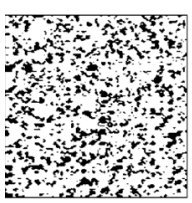

(b1) $\mathrm{G}=1$

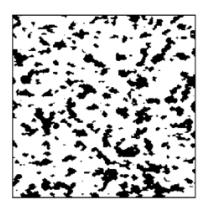

(b2) $\mathrm{G}=2$

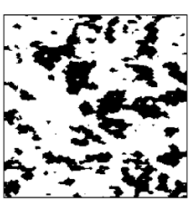

(b3) $\mathrm{G}=3$

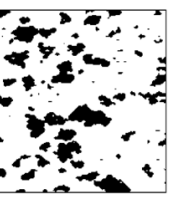

(b7) $\mathrm{G}=7$

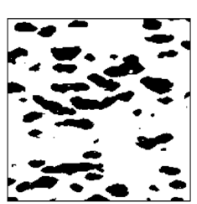

(c3) $\mathrm{G}=3$

(c2) $\mathrm{G}=2$

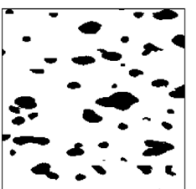

(d2) $\mathrm{G}=2$

(d1) $\mathrm{G}=1$

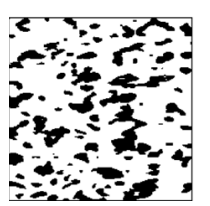

(e1) $\mathrm{G}=1$
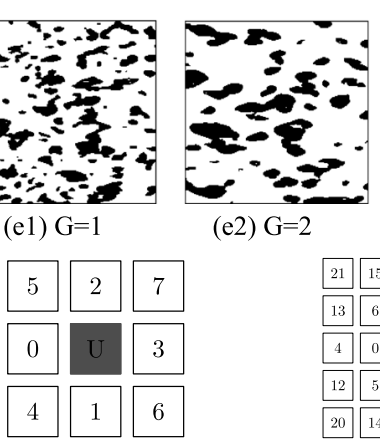

(e2) $\mathrm{G}=2$

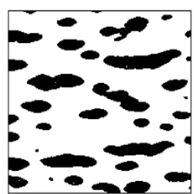

(d3) $\mathrm{G}=3$

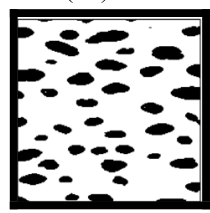

(e3) $\mathrm{G}=3$

\begin{tabular}{|c|c|c|c|c|}
\hline 21 & 15 & 8 & 17 & 23 \\
\hline 13 & 6 & 22 & 10 & 19 \\
\hline 4 & 0 & U & 3 & 11 \\
\hline 12 & 5 & 1 & 9 & 18 \\
\hline 20 & \begin{tabular}{|l|l|}
14 \\
\end{tabular} & 7 & 16 & 22 \\
\hline
\end{tabular}

(f1) template for b1 to b8

(f2) template for $\mathrm{c} 1$ to $\mathrm{c} 6$

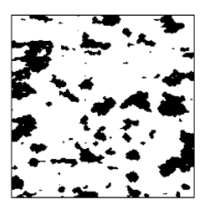

(b4) $\mathrm{G}=4$

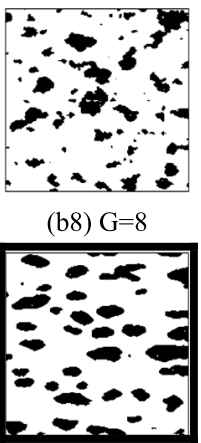

(c4) $\mathrm{G}=4$

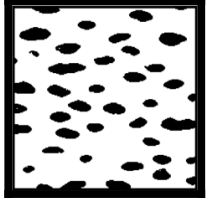

(d4) $\mathrm{G}=4$

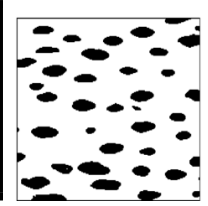

(e4) $\mathrm{G}=4$

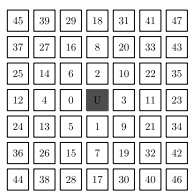

(f3) template for $\mathrm{d} 1$ to $\mathrm{d} 6$

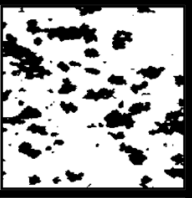

(b5) $\mathrm{G}=5$

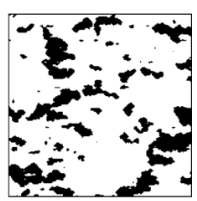

(b6) $\mathrm{G}=6$

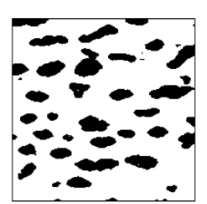

(c5) $\mathrm{G}=5$

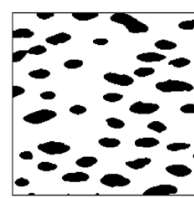

(d5) $\mathrm{G}=5$

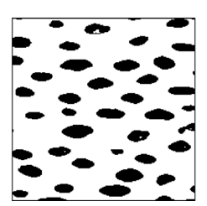

(e5) $\mathrm{G}=5$

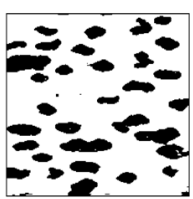

(c6) $\mathrm{G}=6$

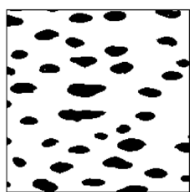

(d6) $\mathrm{G}=6$

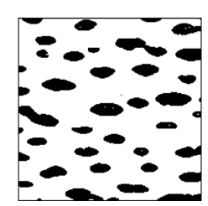

(e6) $\mathrm{G}=6$

Fig. 6. The training image and simulation results of the ellipse-shaped surface objects using data templates with different sizes. The subfigures' captions for (b1)-(e6) are the number of multiple grids used. The subfigures' captions for (f1)-(f4) show the data template used by simulations. The figures highlighted in bold represent the optimal number of multiple-grids.

Fig. 9 shows the plot of NJCS versus the scale of the three training images. The dotted line in the plot corresponds to NJCS $=0.1$. No matter which training image is used, the NJCS fluctuates around 


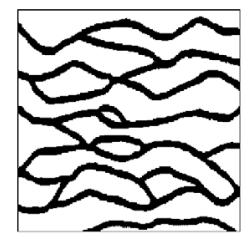

(a) XY-oriented training image. $(250 \times 250$ cells $)$

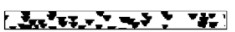

(b) YZ-oriented training image.

$(250 \times 20$ cells $)$

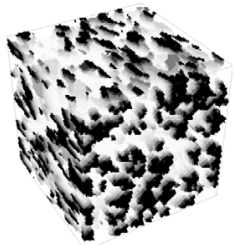

(c1) $\mathrm{G}=1$

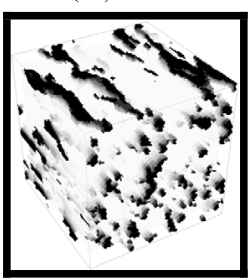

(c4) $\mathrm{G}=4$

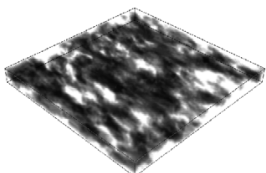

(d1) a section from $\mathrm{c} 1$

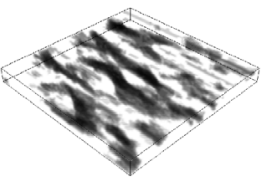

(d4) a section from $\mathrm{c} 4$

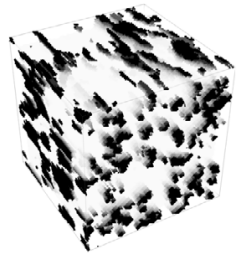

(c2) $G=2$

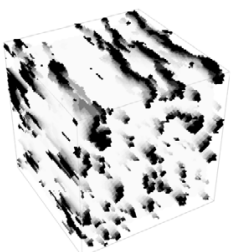

(c5) $\mathrm{G}=5$

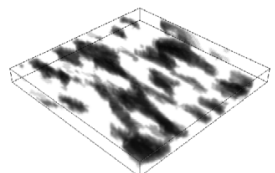

(d2) a section from $\mathrm{c} 2$

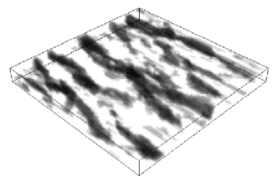

(d5) a section from $\mathrm{c} 5$

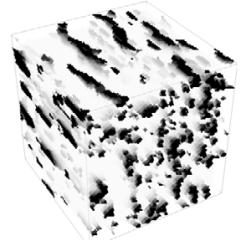

(c3) $\mathrm{G}=3$

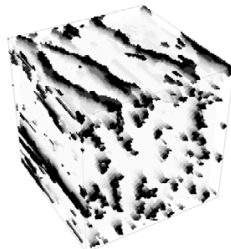

(c6) $\mathrm{G}=6$

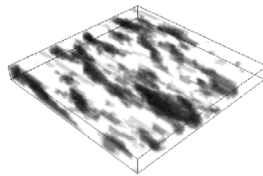

(d3) a section from $\mathrm{c} 3$

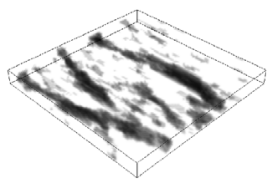

(d6) a section from $\mathrm{c} 6$

Fig. 7. The XY-oriented and YZ-oriented training images for the three-dimensional simulations, and the simulation results using different numbers of multiple grids. The subfigures' captions for (c1)-(c6) are the numbers of multiple grids used. Subfigures (d1)-(d6) are sections from the three-dimensional results (c1)-(c6). The figures highlighted in bold represent the optimal number of multiple-grids.

zero, and almost all NJCSs are smaller than 0.1 when the scale is larger than the target scale. This means that there is little structural information available at larger scales. Moreover, it is necessary to drop structural information at scales larger than the estimated target scale to avoid over-fitting the simulation. Accordingly, the target scale is appropriate for the simulations. 

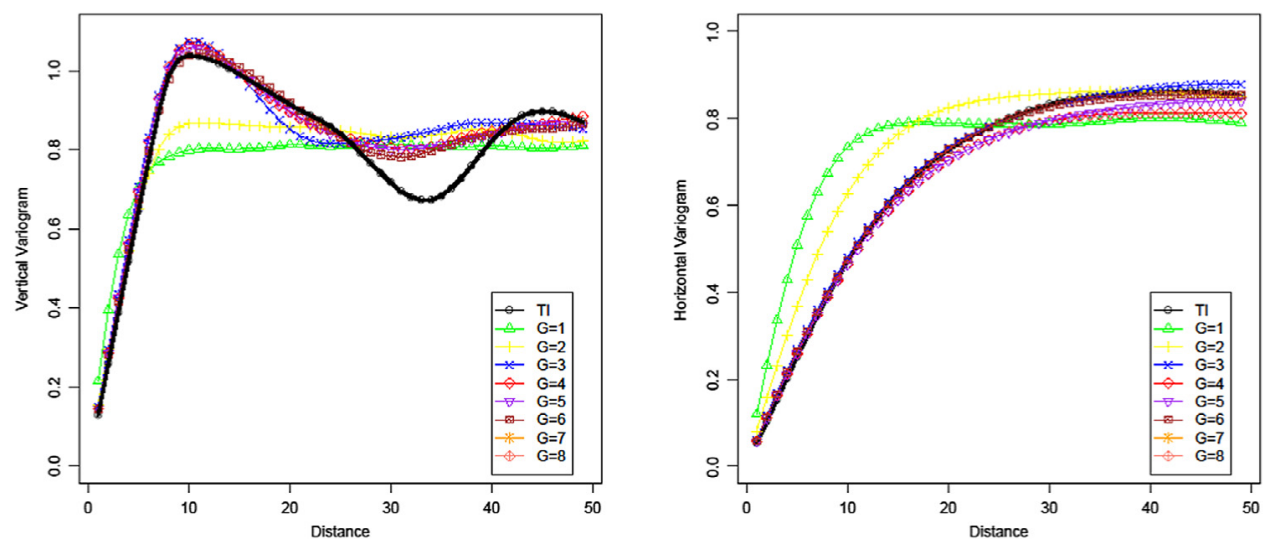

(a) Mean variogram charts using the data template of size 48 .
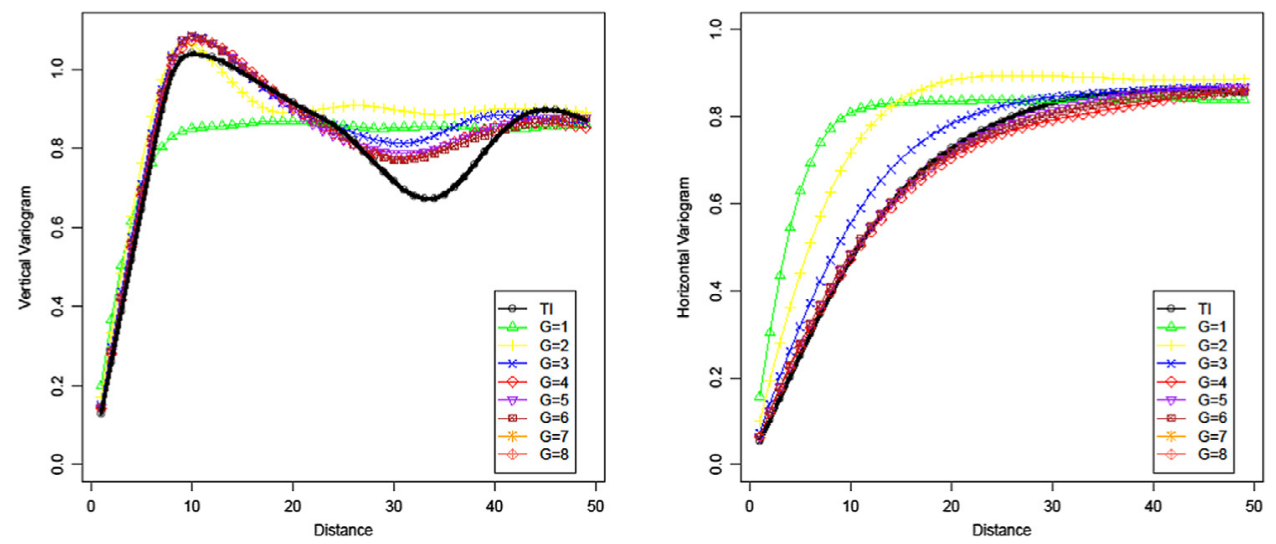

(b) Mean variogram charts using the east-west elongated data template.
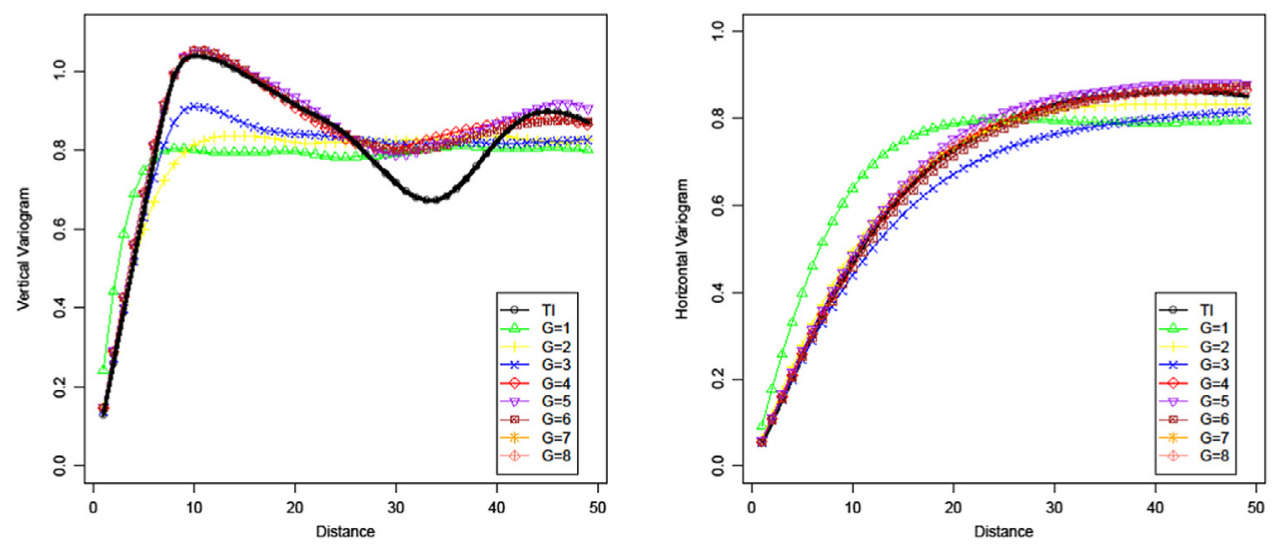

(c) Mean variogram charts using the north-south elongated data template.

Fig. 8. The mean vertical and horizontal variogram charts of the simulation results using the original training image containing curvilinear surface objects and the data template of size 48 , east-west elongated template and the north-south elongated data template. 


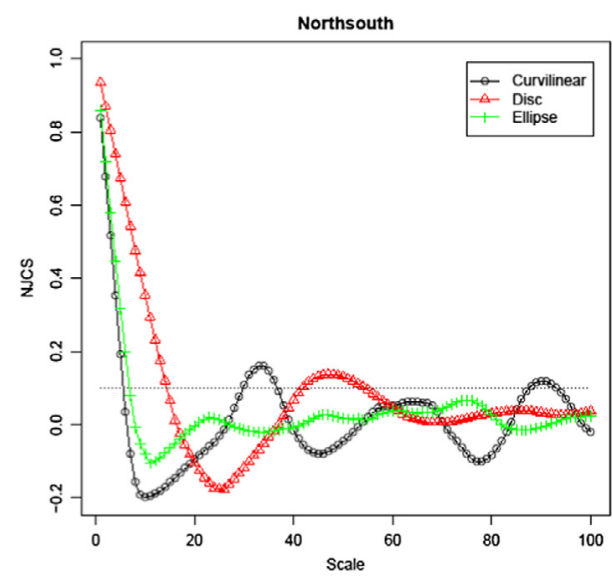

(a) North-south direction.

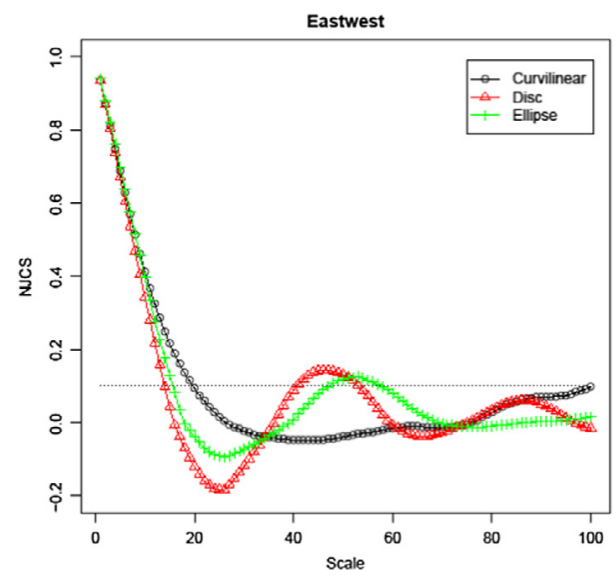

(b) East-west direction.

Fig. 9. The plot of NJCS versus scale of the three training images.

The estimated minimal number of multiple grids is closely related to the geometry and size of the data template used in the simulation. Previous studies (Liu, 2006; Strebelle, 2000) found that the simulation quality was robust to the size and geometric configuration of the data template. However, Strebelle (2000) noted that north-south elongated data templates cannot fully capture long-range continuity in the east-west direction when using a small number of multiple grids.

The proposed method automatically adapts the minimal number of multiple grids according to the data template. Consider the east-west elongated data template (Fig. 2(e)) as an example. $S_{1}^{x}$ changes from 3 to 5 . Because the training image does not change, the target scale $T^{x}$ stays the same. Thus, the number of multiple grids for the $x$-axis is automatically determined by Eq. (2). This ultimately changes the final estimated minimal number of multiple grids. Rows 12 and 13 of Table 1 indicate that the proposed method produced different minimal numbers of multiple grids for the east-west and north-south elongated data templates. The experimental results (Fig. 4(e1)-(f6)) for the elongated data templates also suggest that the simulation quality is insensitive to the elongated data template once the number of multiple grids exceeded the estimated value. The size of the data template also affects the estimation of the minimal number of multiple grids. A large data template can take more neighboring cells into account than a smaller template. However, a larger data template will increase the computation time of building the search tree. Strebelle (2000) recommended that the size of a data template should be no more than 100 . The proposed method can automatically adapt to the data template, regardless of its size. Generally, a smaller data template corresponds to a larger estimated minimal number of multiple grids. This tendency can be seen in Table 1. Moreover, the experimental results (Figs. 5 and 6) using data templates of different sizes demonstrate that the simulation quality does not significantly improve when the number of multiple grids is greater than the estimate, regardless of the data template size.

Increasing the number of grids cannot ensure a high-quality simulation if the data template size is too small to sufficiently reproduce the structural information of the target surface objects. For example, the simulation results that used a data template of size 8 were not acceptable (Fig. 5(b1)-(b8) and Fig. 6(b1)-(b8)), regardless of the number of multiple grids. The quality of the simulation results not only depends on the number of multiple grids used, but also on other parameters used and on the hard data points. The simulation results show that the simulation quality does not improve when the number of multiple grids is larger than that estimated. This shows that the proposed method is effective in estimating the number of multiple grids. To achieve good simulation results, other parameters, such as the template used, the maximum number of conditioning data, and the minimum number of replicas, etc., also should be carefully calibrated in terms of training images and hard data points (Liu, 2006). 
Rescaling the training image changes its grain size (the cell size of the simulation results) (Dungan et al., 2002). For example, the grain size doubles if the training image is rescaled by a factor of 0.5 . However, rescaling the training image does not change its extent (Dungan et al., 2002). Therefore, the estimated number of multiple grids is insensitive to the rescaling of the training image. That is, regardless of the estimated number of multiple grids, the simulation quality improves little when the number of multiple grids is greater than the estimate (see Figs. 3 and 4).

Many MPS algorithms do not use multiple grids, e.g., the direct sampling method (Meerschman et al., 2013). However, these algorithms require a predefined data template or patch size. In such cases, the NJCS can be used to determine the target scale of categorical data. This target scale can be used as the bound of the data template or the patch size, as the spatial structure information is negligible at scales larger than the target scale.

The proposed method estimates the minimal number of multiple grids needed from the perspective of considering enough spatial association during simulation. The advantage of our method is that it can estimate a minimal number of multiple grids that can consider sufficient spatial association through setting the threshold for NJCS on an application-dependent basis. This gives a criterion for a minimal number of multiple grids. Some researchers have used correlation functions, which is closely related to the variogram, to set the scale of target surface objects, for example, Kolbjørnsen et al. (2014). However, they also pointed out that the multiple grid number must be set manually. Meanwhile, the correlation should be calculated several times for training images with multiple categories. The NJCS could take into account all the categories simultaneously. At last, the method proposed frames the question of setting the minimal number of multiple grids as a question of how much spatial associations between objects should be taken into account during the simulations.

Besides correlation functions, another candidate is the indicator variogram. Compared with indicator variograms, there are two advantages of using the NJCS of all categories. First, the indicator variogram can only be applied to two category cases. For multiple category cases, indicator variograms should be calculated for each category and the other categories should be merged. Accordingly, our method needs only a single scan of the training image, whereas indicator variograms need $M$ scans of the training image with $\mathrm{M}$ being the number of categories in the training image. Obviously, the indicator variogram needs more computation resources than the method proposed. Second, when the indicator variogram is used for multiple category cases, it only considers the relation between one category and all other categories. It may underestimate the coarsest scale for this category. For example, when the current category is highly correlated with a category that has a small marginal probability, this correlation may be diluted by other categories as all these categories are merged into one category. Compared with the indicator variogram, the NJCS does not merge any category and can take into account all inter-category relations simultaneously.

The proposed method is sensitive to the degree of spatial associations between objects that should be considered during the simulations. This degree, i.e., the NJCS threshold used in the experiment is set subjectively by the user. Generally, the smaller the threshold the larger the number of multiple grids. Meanwhile, the minimal number of multiple grids is also closely related to the training image used. Besides experts experience, a plot of the relation between the NJCS value and the scale is helpful in determining the NJCS threshold used. Furthermore, the NJCS parameter is worthy of a sensitivity analysis to achieve acceptable simulation results for future researches and applications.

\section{Conclusion}

This paper has described a quantitative method for estimating the minimal number of multiple grids for SNESIM. The method uses spatial association analysis as a tool to find the largest-scale structural information to be considered in a simulation. The minimal number of multiple grids is estimated using the criterion that the simulation should consider the largest-scale structural information that is statistically significant in the training image. Two-dimensional and threedimensional experimental results showed that simulations using the estimated minimal number of multiple grids generate reasonable results, and that increasing the number of multiple grids did not significantly improve the quality. These experiments have demonstrated that the proposed method adapts to the geometric structure of the target surface objects, the data template configuration, and 
the size of the training image when the training image accurately represents the large-scale structural information of the target surface objects.

In future work, the basic idea and framework of the proposed method will be extended to other MPS algorithms using appropriate measures of spatial structural information. For example, to simulate continuous variables, Moran's I (Moran, 1950) or a variogram could be used to measure spatial associations in estimating the target scale of training images. The spatial association can also help determining the most appropriate data template for simulation. For example, what is the minimal span of a rectangular template, i.e., for any data template contains the estimated minimal rectangular template, a large multiple grid number can ensure acceptable simulation results.

Our method estimates the minimal multiple grid number from the perspective of spatial association. There should be other criteria, for example the spatial heterogeneity and measures for complex spatial patterns, to be used to help setting the multiple grid number. We will study this issue in our future work.

Furthermore, the size of the data template could also be optimized according to the minimum structure size in the training image. A good data template should simultaneously consider the computation time, the simulation quality and the resulting uncertainty space. Moreover, for highdimensional simulations, there may be large differences in the estimated minimal number of multiple grids for different axes. A solution could be to use an adaptive data template, which may further reduce the computation time and increase the diversity of simulated patterns. In this case, the relationship between the minimal number of multiple grids and the target scale of training images will become more complicated.

\section{Acknowledgments}

This study was supported by the Natural Science Foundation of China (Nos. 41101440, 41471296), Youth Foundation of Shanxi Province (No. 2012021015-1), and China Postdoctoral Science Foundation (2013M530891).

\section{References}

Bai, H., Ge, Y., 2015. Determining the spatial scale that multi-point simulations should consider using spatial association analysis: using snesim as an example. In: The 17th Annual Conference of the International Association for Mathematical Geosciences. Freiberg, Saxony, Germany, (in press).

Bai, H., Li, D., Ge, Y., Wang, J.F., 2016. Detecting nominal variables' spatial associations using conditional probabilities of neighboring surface objects' categories. Inform. Sci. 329, 701-718. http://dx.doi.org/10.1016/j.ins.2015.10.003.

Boucher, A., 2008. Super resolution mapping with multiple point geostatistics. In: Soares, A., Pereira, M.J., Dimitrakopoulos, M. (Eds.), geoENV VI -Geostatistics for Environmental Applications. Springer, Netherlands, pp. 297-305. http://dx.doi.org/10. 1007/978-1-4020-6448-7_25.

Boucher, A., 2009. Considering complex training images with search tree partitioning. Comput. Geosci. 35, 1151-1158. http://dx.doi.org/10.1016/j.cageo.2008.03.011.

Cears, J., Zhang, T., 2004. Multiple-point geostatistics: a quantitative vehicle for integrating geologic analogs into multiple reservoir models. In: Grammer, G.M., Harris, P.M., Eberli, G.P. (Eds.), Integration of Outcrop and Modern Analogs in Re Servoir Modeling: AAPG Memoir. Vol. 80. American Association of Petroleum Geologists, Tulsa, pp. 13-15.

Cliff, A.D., Ord, J.K., 1970. Spatial autocorrelation: A review of existing and new measures with applications. Econ. Geogr. 46, 269-292. http://dx.doi.org/10.2307/143144.

Cliff, A.D., Ord, J.K., 1981. Spatial Processes: Models and Applications. Pion Ltd., London, UK.

Comunian, A., Renard, P., Straubhaar, J., 2012. 3D multiple-point statistics simulation using 2D training images. Comput. Geosci. 40, 49-65. http://dx.doi.org/10.1016/j.cageo.2011.07.009.

Dale, M.R.T., Fortin, M.J., 2014. Spatial Analysis: A Guide for Ecologists. Cambridge University Press, Cambridge, United Kingdom.

Dungan, J.L., Perry, J.N., Dale, M.R.T., Legendre, P., Citron-Pousty, S., Fortin, M.J., Jakomulska, A., Miriti, M., Rosenberg, M.S., 2002. A balanced view of scale in spatial statistical analysis. Ecography 25, 626-640. http://dx.doi.org/10.1034/j.16000587.2002.250510.x.

Ge, Y., 2013. Sub-pixel land-cover mapping with improved fraction images upon multiple-point simulation. Int. J. Appl. Earth Obs. 22, 115-126. http://dx.doi.org/10.1016/j.jag.2012.04.013.

Ge, Y., Bai, H., 2011. Multiple-point simulation-based method for extraction of objects with spatial structure from remotely sensed imagery. Int. J. Remote Sens. 32, 2311-2335. http://dx.doi.org/10.1080/01431161003698278.

Guardiano, F., Srivastava, R., 1993. Multivariate geostatistics: beyond bivariate moments. In: Soares, A. (Ed.), Geostatistics-Troia. Kluwer Academic, Dordrecht, pp. 133-144.

Hajizadeh, A., Safekordi, A., Farhadpour, F.A., 2011. A multiple-point statistics algorithm for 3D pore space reconstruction from 2D images. Adv. Water Resour. 34, 1256-1267. http://dx.doi.org/10.1016/j.advwatres.2011.06.003. 
Honarkhah, M., Caers, J., 2010. Stochastic simulation of patterns using distance-based pattern modeling. Math. Geosci. 42, $487-517$.

Hovadik, J.M., Larue, D.K., 2007. Static characterizations of reservoirs: refining the concepts of connectivity and continuity. Petrol. Geosci. 13, 195-211.

Jha, S.K., Mariethoz, G., Evans, J.P., McCabe, M.F., 2013. Demonstration of a geostatistical approach to physically consistent downscaling of climate modeling simulations. Water Resour. Res. 49, 245-259.

Journel, A., 1993. Geostatistics: roadblocks and challenges. Math. Geosci. 92, 213-224. http://dx.doi.org/10.1007/978-94-0111739-518.

Journal, A., 2000. Stochastic imaging of channels from seismic data using multiple point statistics. In Report 13. Stanford Center for Reservoir Forcasting, Stanford, CA.

Kolbjørnsen, O., Stien, M., Kjønsberg, H., Fjellvoll, B., Abrahamsen, P., 2014. Using multiple grids in Markov mesh facies modeling. Math. Geosci. 46, 205-225. http://dx.doi.org/10.1007/s11004-013-9499-5.

Leslie, T.F., Kronenfeld, B.J., 2011. The colocation quotient: a new measure of spatial association between categorical subsets of points. Geogr. Anal. 43, 306-326. http://dx.doi.org/10.1111/j.1538-4632.2011.00821.x.

Li, H., Arpat, B.G., Caers, J., 2005. A new multiple grid method in simpat: a proposal. In Report 18. Stanford Center for Reservoir Forcasting, Stanford, CA.

Liu, Y., 2006. Using the snesim program for multiple-point statistical simulation. Comput. Geosci. 32, 1544-1563. http://dx.doi.org/10.1016/j.cageo.2006.02.008.

Mahmud, K., Mariethoz, G., Baker, A., Sharma, A., 2015. Integrating multiple scales of hydraulic conductivity measurements in training image-based stochastic models. Water Resour. Res. 51, 465-480. http://dx.doi.org/10.1002/2014WR016150.

Mariethoz, G., Lefebvre, S., 2014. Bridges between multiple-point geostatistics and texture synthesis: Review and guidelines for future research. Comput. Geosci. 66, 66-80. http://dx.doi.org/10.1016/j.cageo.2014.01.001.

Mariethoz, G., Philippe, R., Julien, S., 2011. Extrapolating the fractal characteristics of an image using scale-invariant multiplepoint statistics. Math. Geosci. 43, 783-797. http://dx.doi.org/10.1007/s11004-011-9362-5.

Meerschman, E., Pirot, G., Mariethoz, G., Straubhaar, J., Van Meirvenne, M., Renard, P., 2013. A practical guide to performing multiple-point statistical simulations with the direct sampling algorithm. Comput. Geosci. 52, $307-324$. http://dx.doi.org/10.1016/j.cageo.2012.09.019.

Meerschman, E., Van Meirvenne, M., Mariethoz, G., Islam, M.M., De Smedt, P., Van De Vijver, E., Saey, T., 2014. Using bivariate multiple-point statistics and proximal soil sensor data to map fossil ice-wedge polygons. Geoderma 213, 571-577. http://dx.doi.org/10.1016/j.geoderma.2013.01.016.

Mirowski, P., Tetzlaff, D., Davies, R., McCormick, D., Williams, N., Signer, C., 2009. Stationarity scores on training images for multipoint geostatistics. Math. Geosci. 41, 447-474. http://dx.doi.org/10.1007/s11004-008-9194-0.

Moran, P.A.P., 1950. Notes on continuous stochastic phenomena. Biometrika 37, 17-23. http://dx.doi.org/10.2307/2332142.

Okabe, H., Blunt, M.J., 2004. Prediction of permeability for porous media reconstructed using multiple-point statistics. Phys. Rev. E 70, 066135. http://dx.doi.org/10.1103/PhysRevE.70.066135.

Pérez, C., Mariethoz, G., Ortiz, J.M., 2014. Verifying the high-order consistency of training images with data for multiple-point geostatistics. Comput. Geosci. 70, 190-205. http://dx.doi.org/10.1016/j.cageo.2014.06.001.

Remy, N., Boucher, A., Wu, J., 2009. Applied Geostatistics with SGeMS: A User's Guide. Cambridge University Press, Cambridge.

Renard, P., Allard, D., 2013. Connectivity metrics for subsurface flow and transport. Adv. Water Resour. 51, 168-196.

Straubhaar, J., Renard, P., Mariethoz, G., Froidevaux, R., Besson, O., 2011. An improved parallel multiple-point algorithm using a list approach. Math. Geosci. 43, 305-328.

Strebelle, S., 2000. Sequential simulation drawing structures from training images (Ph.D. thesis), Stanford University, Stanford, CA, USA.

Strebelle, S., 2002. Conditional simulation of complex geological structures using multiple-point statistics. Math. Geosci. 34, 1-21. http://dx.doi.org/10.1023/A:1014009426274.

Tahmasebi, P., Hezarkhani, A., Sahimi, M., 2012. Multiple-point geostatistical modeling based on the cross-correlation functions. Comput. Geosci. 16, 779-797. http://dx.doi.org/10.1007/s10596-012-9287-1.

Tang, Y., Atkinson, P.M., Wardrop, N.A., Zhang, J., 2013. Multiple-point geostatistical simulation for post-processing a remotely sensed land cover classification. Spat. Stat. 5, 69-84. http://dx.doi.org/10.1016/j.spasta.2013.04.005.

Xu, Z., Teng, Q., He, X., Yang, X., Li, Z., 2012. Multiple-point statistics method based on array structure for 3D reconstruction of Fontainebleau sandstone. J. Pet. Sci. Eng. 100, 71-80. http://dx.doi.org/10.1016/j.petrol.2012.11.005.

Zhang, T., Switzer, P., Journel, A., 2005. Merging prior structural interpretation and local data: The Bayes updating of multiplepoint statistics. In: Cheng Q. Bonham-Carter G. (Eds.), Proceeding of International Association for Mathematical Geology, Toronto, Canada, pp. 615-620.

Zhang, T., Switzer, P., Journel, A., 2006. Filter-based classification of training image patterns for spatial simulation. Math. Geosci. 38, 63-80. http://dx.doi.org/10.1007/s11004-005-9004-x. 